MATEC Web of Conferences 42, 01010 (2016)

DOI: $10.1051 /$ matecconf/20164201010

(C) Owned by the authors, published by EDP Sciences, 2016

\title{
A New Fault Simulation Test and Evaluation Method of Distribution Automation System
}

\author{
Wenbo Fan ${ }^{1}, \mathrm{Yu} \mathrm{An^{2 }}$, Dong Liü ${ }^{2, a}$ and Shilei Guan ${ }^{1}$ \\ ${ }^{1}$ China Electric Power Research Institute, Beijing, China \\ ${ }^{2}$ Shanghai Jiao Tong University, Shanghai, China
}

\begin{abstract}
This paper proposes a fault simulation test method suitable for five divisions of power supply area. Distribution network structures are classified according to different power supply areas and more active interference is injected into test cases, which makes fault scenarios more diverse. Besides, to evaluate the whole process of fault isolation and recovery, the index of fault isolation and index of fault restoration are presented separately, and power recovery rate is provided to evaluate the load transfer capability. In the end, two typical test cases are displayed to clarify the whole test procedure and the use of evaluation method.
\end{abstract}

\section{Introduction}

Distribution automation is an important part of implementation of smart distribution network. The field operation of the distribution automation system (DAS) can improve the quality and reliability of power supply; greatly advance the management level of power dispatch and operation [1-3]. When a fault occurs, DAS should be able to accomplish automatic fault location, isolation and restoration of non-fault area in a short time. Hence, in order to ensure reliable operation and effective utilization of power dispatching, a test scheme of DAS needs to be provided before the system put into practical use.

In the early stage, due to lack of test method, the functions of DAS could only be tested in a long-term operation, which leads to some problems exposing too late to be solved and causes the waste of facility resources. Later, direct testing method is proposed. This method allows direct testing on the running network and then checks the normal monitoring capability and fault processing functions. But direct testing has the potential to cause serious damage to distribution network because electrical connection between distribution lines becomes increasing close. The simulation and test technology of DAS, therefore, gradually developed [4-7].

Current research achievements of fault simulation test are comparatively few. A set of feeder automation simulation test environment is established based on parallel computing theory [8], which gives valuable attempt to testing on fault processing function of DAS. Some domestic researchers has put forward a host injection testing method [9], which is capable of simulating various faults on the feeders to test the feeder automation behavior of DAS, but lack of the evaluation on the result of fault processing. Reference [10] presents a failure-free evaluation test method for feeder fault location, isolation and restoration algorithm of reliability. But it's more an algorithm evaluation than a function evaluation. In [11], a time correlation index is used as the reliability index to evaluate DAS, but also, lack of fault processing function evaluation.

From the above, current fault simulation test system is relatively single in system operation mode and fault scenario design, lacking practical significance on field operation testing. When fault processing function involved, it is incapable of logic test for more complicated distribution network. Meanwhile, it is also necessary to set up the index system for fault processing function. To add more practical fault scenarios, a fault simulation test method suitable for five divisions of power supply area is proposed in this paper, and a treatment effect evaluation standard based on protection operating process is also established.

\section{Distribution network classification and fault scenario design}

\subsection{Five Divisions of Power Supply Areas}

In order to make test case more close to the practical operation of distribution network, this section will classify all kinds of typical distribution network and design a complete fault testing scenarios based on five divisions of power supply area.

With reference to the national grid company enterprise standard Q/GDW 732-2012, the so-called five divisions of power supply area, are mainly on the basis of administrative levels, as in Fig. 1. When there are multiple choices, it can be determined comprehensively

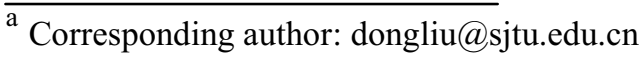


with load density (Table 1), user importance, electricity consumption, GDP and other factors.

Table 1. Load density reference for power supply area.

\begin{tabular}{|c|c|}
\hline Power supply area & $\begin{array}{c}\text { Reference range for load } \\
\text { density } \boldsymbol{\sigma}\left(\mathbf{k W} / \mathbf{k m}^{2}\right)\end{array}$ \\
\hline A & $\sigma \geqslant 20000$ \\
\hline B & $6000 \leqslant \sigma<20000$ \\
\hline C & $1000 \leqslant \sigma<6000$ \\
\hline D & $100 \leqslant \sigma<1000$ \\
\hline E & $\sigma<100$ \\
\hline
\end{tabular}

In Fig. 1, urban district, down town, towns and rural are defined as follows:

\section{1) Urban District}

Area that is municipality directly under the Central Government or prefecture-level city.

2) Down Town

Populated area where administration, economy, commerce and traffic are centralized.

3) Towns

Area where industry and population are relatively concentrated.

4) Rural

Area takes agriculture as the dominant.

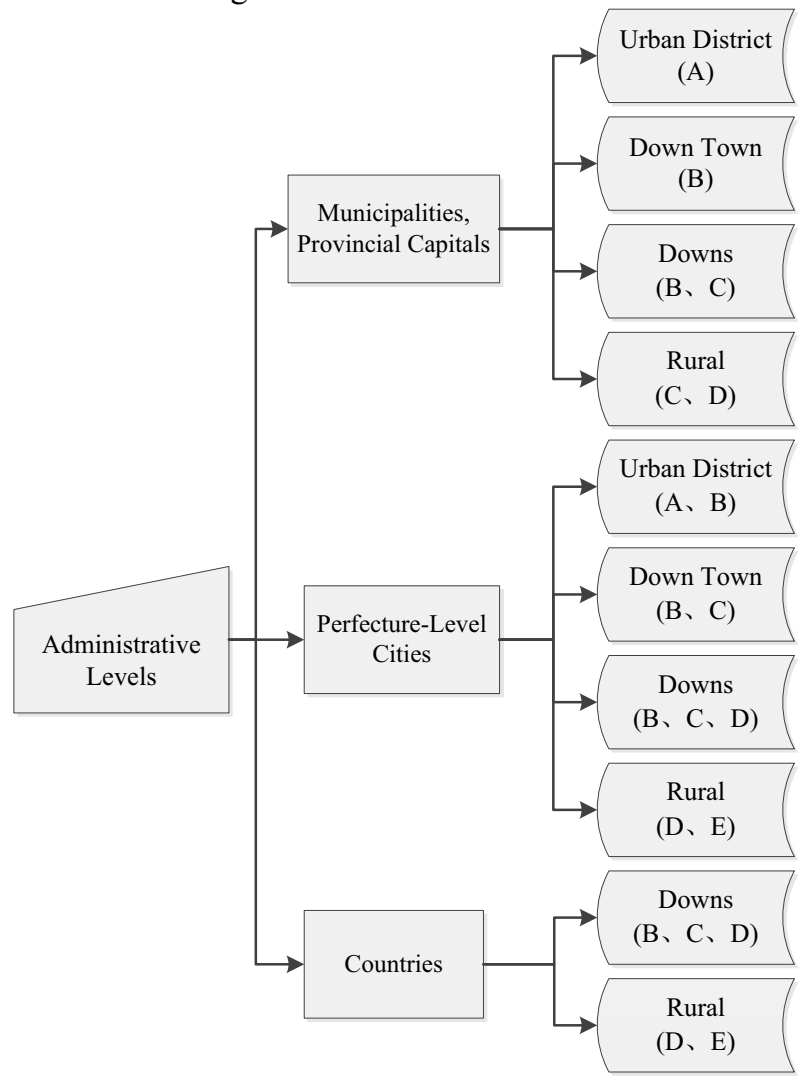

Figure 1. Divisions of power supply area.

For $10 \mathrm{kV}$ distribution line construction standards, areas of low load density ( $\mathrm{D}$ and $\mathrm{E}$ ) should be given priority to with overhead lines; areas of medium load density (B and $\mathrm{C}$ ) should also be given priority to with overhead lines, if necessary, use cable connection; areas of high load density (A) should be given priority to with cables supplemented by overhead lines. Typical $10 \mathrm{kV}$ distribution network should include radial configuration, multi-source network, multi-sectioned and multi-linked network and other loop networks. And classification of these typical distribution networks according to the divisions of power supply area is as shown in Table 2 .

In Table 2, almost all of $10 \mathrm{kV}$ distribution network types are covered and classified into different divisions of power supply area, which would better suit practical need of fault simulation testing. During the DAS test, firstly determine the type of power supply area of distribution network that needs to be tested, and secondly choose the typical distribution networks of this kind of power supply area for testing.

Table 2. Classification of $10 \mathrm{kV}$ typical distribution network.

\begin{tabular}{|c|c|c|c|}
\hline $\begin{array}{l}\text { Power Supply } \\
\text { Area }\end{array}$ & \multicolumn{3}{|c|}{$\begin{array}{c}\text { Classification of } 10 \mathrm{kV} \text { typical distribution } \\
\text { network structures }\end{array}$} \\
\hline \multirow[t]{2}{*}{ A } & Cable & & $\begin{array}{l}\text { Double loop network } \\
\text { Bijection network } \\
\text { Three double network } \\
\text { Two power supply with } \\
\text { one backup network } \\
\text { Petal shaped network }\end{array}$ \\
\hline & Overhead & & $\begin{array}{l}\text { Three-sectioned and three- } \\
\text { linked network } \\
\text { Six power supply network }\end{array}$ \\
\hline \multirow{2}{*}{ B } & Cable & & $\begin{array}{l}\text { Two-sectioned and two- } \\
\text { linked network } \\
4 * 6 \text { network }\end{array}$ \\
\hline & Overhead & & $\begin{array}{l}\text { Monocyclic rings contact } \\
\text { network } \\
\text { Composite ring network }\end{array}$ \\
\hline \multirow{2}{*}{$\mathrm{C}$} & Cable & & $\begin{array}{l}\text { Two contact network } \\
\text { Three contact network }\end{array}$ \\
\hline & Overhead & 1) & Monocyclic ring network \\
\hline $\mathrm{D}$ & Overhead & 1) & Monocyclic ring network \\
\hline $\mathrm{E}$ & Overhead & & $\begin{array}{l}\text { Tree network } \\
\text { Chain network }\end{array}$ \\
\hline
\end{tabular}

\subsection{Fault scenario design}

In China, most of MV networks are neutral nongrounding or neutral earthing via arc system. So the faults are mainly divided into three-phase grounding fault, inter phase short circuit fault, two-phase grounding fault and single-phase grounding fault [12]. Besides short circuit fault, some active interferences should be taken into account, such as misinformation and refusal-operation of telesignalisation, signal shielding, breaker locking, etc.

And these active interferences may occur in many reasons:

\section{1) Equipment}

Terminal quits operation due to self-fault; equipment cannot be put into operation and need s maintenance due to aging; parameter configuration error of master station and abnormal program, etc.

2) Communication 
The wireless signal blind area, signal shielding or message channel failure lead to problems such as data communication breakdown; communication channel is too narrow to incomplete data transmission and lose information; switch misoperation or remote control failure due to communication error in the process of fault handling; switch rejecting act because of signal interference or network outages; accidental mistakes caused by deviation of time synchronization system in terminals, etc.

\section{3) Environment}

Urban construction forces terminals to quit; telesignalling misoperation caused by voltage fluctuation; junction oxidation leads to information jitter, etc.

\section{4) Manual operation}

Not dispatch terminal operation mode switch to maintenance state during the debugging of master station function; local manual operation without permit of the scheduling, etc.

To sum up, considering so many kinds of DAS control mode, such as existing centralized master station control, distributed area control and local control, when designing typical fault scenarios, it not only needs to consider the classification of fault location and fault type, but also takes more active interference and operation condition into account, in order to ensure the diversity and extensibility of test cases. Moreover, the individual test case should allow a variety of fault scenarios to occur simultaneously. Typical distribution network fault scenarios are summarized in Table 3.

Table 3. Font styles for a reference to a journal article.

\begin{tabular}{|c|ll|}
\hline Category & \multicolumn{2}{|c|}{ Fault Scenario } \\
\hline Location of fault & 1) & Main road \\
& 2) & Branch \\
\hline \multirow{3}{*}{ Fault type } & 1) & Grounding fault (single- phase \\
& two-phase / three-phase) \\
& 2) & Inter phase fault \\
\hline \multirow{5}{*}{ Active interference } & 1) & Switch rejecting act \\
& 2) & Misoperation of switch \\
& 3) & Switch delay response \\
& 4) & Communication interrupt \\
& 5) & Lockout \\
\hline Operation situation & 1) & Opening loop operation \\
& 2) & Load Transfer Margin \\
& 3) & Line maintenance \\
4) & Backup line fault \\
\hline \multirow{3}{*}{ Multi-fault } & 1) & Opening loop operation \\
& 2) & Load Transfer Margin \\
& 3) & Line maintenance \\
4) & Backup line fault \\
\hline
\end{tabular}

\section{Evaluation method for fault processing function}

Evaluation of test results of DAS fault processing function depends on two aspects: the process and the result of protection after faults. Correct results may discover some hidden action errors, which may lead to the expansion of fault area during the fault processing.

Different vendors in fault handling use different strategies and control modes. But all the strategies must follow two stages: fault isolation and fault restoration. A sign to differ two stages is whether there is short circuit current in the distribution network. Unless short circuit current does not exist in the network, the system should not enter the stage of fault restoration.

\subsection{Index for fault isolation function}

The evaluation index of fault isolation function is relative clear. In the stage of fault isolation, all of fault line switches should be disconnected, and non-fault line switches should not occur false tripping. Because the false tripping from non-fault line switches may lead to the expansion of fault area. Two conditions are discussed separately:

1)Tie Switch on the Fault Line

In this situation, tie switch is normally in the OFF status and should not need to be triggered.

\section{2) No Tie Switch on the Fault Line}

In this situation, all the switches on the fault line should be opened when in fault isolation process.

This paper will propose index $K_{A}$, index $K_{l}$ and index $K_{2}$ for evaluation of fault isolation. The evaluation rules are as shown in Table 4.

Table 4. Indexes for fault isolation function.

\begin{tabular}{|c|c|c|}
\hline Index & Situations & Value \\
\hline \multirow{2}{*}{$K_{1}$} & Fault line switches are all in the OFF status & 1 \\
\cline { 2 - 3 } & Any fault line switch is in the ON status & -1 \\
\hline \multirow{2}{*}{$K_{2}$} & Switch misoperation on the non-fault line & 1 \\
\cline { 2 - 3 } & No action for non-fault line switches & 0 \\
\hline
\end{tabular}

A discriminant is constructed as follow:

$$
K_{A}=K_{1}-K_{2}
$$

Index $K_{A}>0$ indicates correct fault isolation, while any other value of index $K_{A}$ indicates an error occurred during fault processing.

\subsection{Index for fault restoration function}

When there is no short circuit current in circuit lines and index $K_{A}>0$, it indicates DAS has finished fault isolation correctly and starts to enter the restoration stage. The aim of fault restoration is to close tie switch and restore the normal power supply of non-fault area.

Make sure that tie switch is not on the fault line before closing tie switch. The misoperation of fault line switches will further cause the short-circuit fault in other areas. The index KB is used to evaluate the misoperation of fault line switches. Index $K_{B}=-1$ indicates misoperation, while index $K_{B}=1$ indicates no action on fault line. And the initial value of KB is 1 . To monitor the value of index $K_{B}$ during fault restoration: index $K_{B}<0$ indicates failure while index $K_{B}>0$ indicates success of fault restoration. Power recovery rate SR can be obtained from the following formula, where $P_{A}$ is the restoration of power supply per unit time and $P_{B}$ is the power supply in normal operation per unit time. 


$$
S_{R}=P_{A} / P_{B} \times 100 \%
$$

Obviously, power recovery rate $S_{R}$ is associated with the length of unit time. The longer the unit time, the less the comparability of the index is. Considering most of fault processing time is within three minutes, we take three minutes as unit time.

\section{Test procedure}

In this paper, the fault simulation test process is completed in five steps..

Step 1: According to the section needs to be tested by DAS, combined with load density, user importance, electricity consumption levels, and other factors such as GDP, determine the power supply area and choose the typical distribution network structures in this kind of power supply area for testing.

Step 2: After determining the distribution network structures start to establish distribution network model. Configure the device table for acquisition device simulation on field terminals; configure the simulation channel for all kinds of switches; choose the type of communication and communication parameters; configure the switch parameters and load parameters; draw the map of distribution network; connect the map with database, and generate topology information in the database.

Step 3: Designing the test case. Combined with the control mode of DAS, design the corresponding fault scenarios, and generate orderly test cases. Add more active interferences into test case when DAS is of distributed control mode.

Step 4: Test cases simulation. Inject fault information into network and rebuild distribution network topology analysis. By collecting switch parameters and other electrical data, FA system completes the logic analysis, transmits the remote control command, and accomplishes fault isolation and restoration.

Step 5: Evaluate DAS fault processing efficiency according to the generated test report. Index $K_{A}$ evaluate the process of fault isolation while index $K_{B}$ evaluates the process of fault restoration. In the end, the test report can provide power recovery rate $S_{R}$, which evaluate the load transfer capability.

\section{Test cases}

In this part, two typical test cases will be displayed. For example, if a cable network with single tie switch needs to be tested by DAS, after modeling and test cases design, start simulation and all the test cases will implement in order.

\subsection{Switch rejecting action}

In this case, short-circuit fault and communication interrupt are considered at same time.

Simulation starts and the switch L4 with a yellow dot is suffered in communication failure, as shown in Fig. 2(a). The short-circuit fault takes place between switch
L3 and switch L4 as designed in advance. In Fig. 2(b), because of communication failure, the switch L4 has no action but the opposite switch L3 separate the fault line correctly.

Use Table. 4 to evaluate the efficiency of fault processing. The switch on fault line has no action, $K_{l}=-1$; there are no switches misoperating on the non-fault line, $K_{2}=0$. Refer to "(1)", $K_{A}<0$, which indicate potential safety hazard. Although tie switch L5 remains OFF status and avoid the extending of fault section to some extent, but it should not be regarded as a successful fault processing.

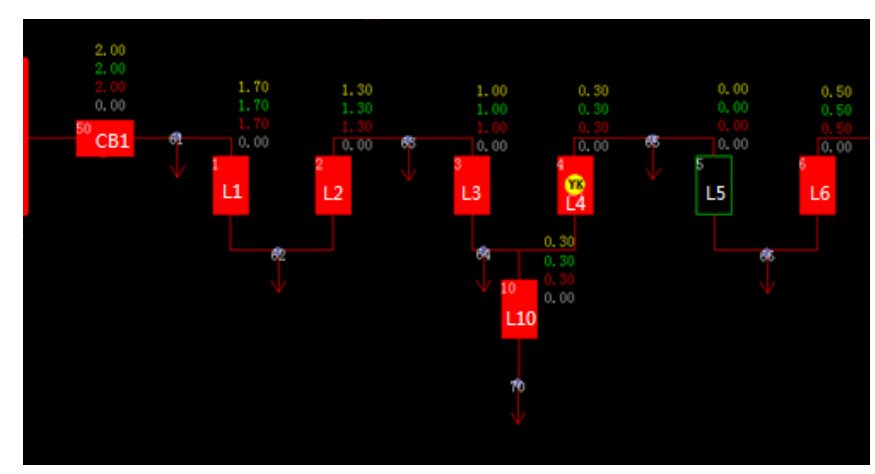

(a) Communication failure at L4.

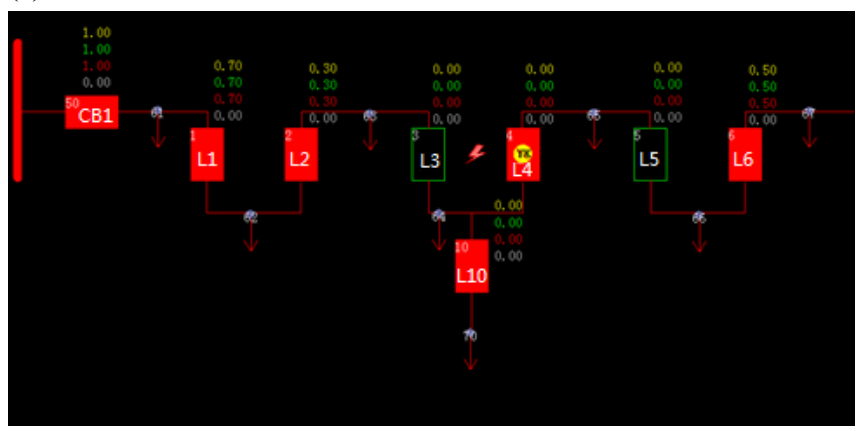

(b) L4 rejects action.

Figure 2. Switch rejecting action.

\subsection{Load transfer margin case}

In sufficient load transfer margin case, as shown in Fig. 3, the fault takes place between switch L1 and switch L2. Circuit breaker CB1 occurs tripping at first in (a), and secondly the switches on the fault line open in (b). Finally tie switch L5 close and the power supply of loss load is transferred to the opposite substation in (c). To evaluate the fault processing efficiency, $K_{1}=1$ and $K_{2}=0$ leads to $K_{A}>0$, which indicate correct fault isolation function. At fault restoration stage, switches on the fault line have no misoperation, $K_{B}=1>0$, which indicate no errors during fault restoration. Therefore, considering fault isolation and restoration in two stages, the fault treatment is a success.

For insufficient load transfer margin case in Fig. 4, change the load value to exceed the size of load transfer margin, and the tie switch remains OFF status after fault isolation. The stage of fault isolation is successful and there are no switches occurs misoperation during the stage of fault restoration, which indicate correct fault restoration function. In addition, although some load of non-fault area fails to restore power supply, it is an issue 
for design and planning of power system, not the error in fault processing. So in this place, the whole process is safe and correct.

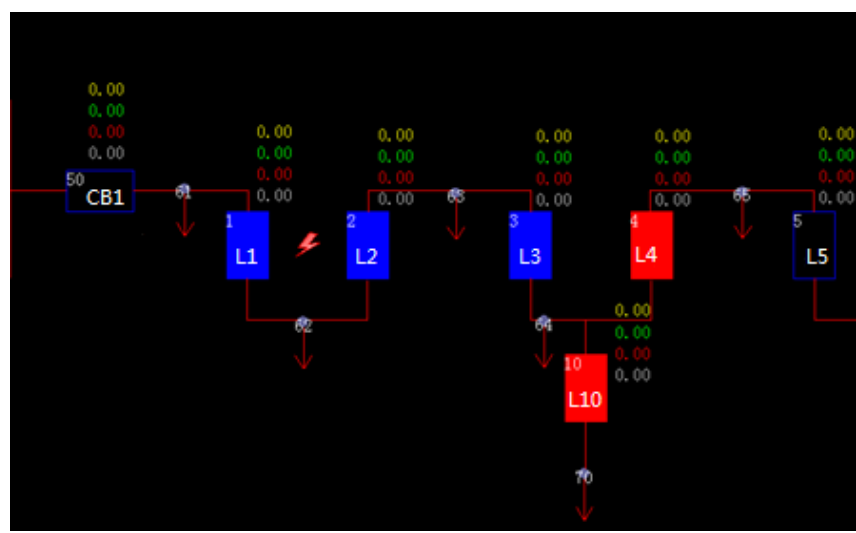

(a) Fault occurs.

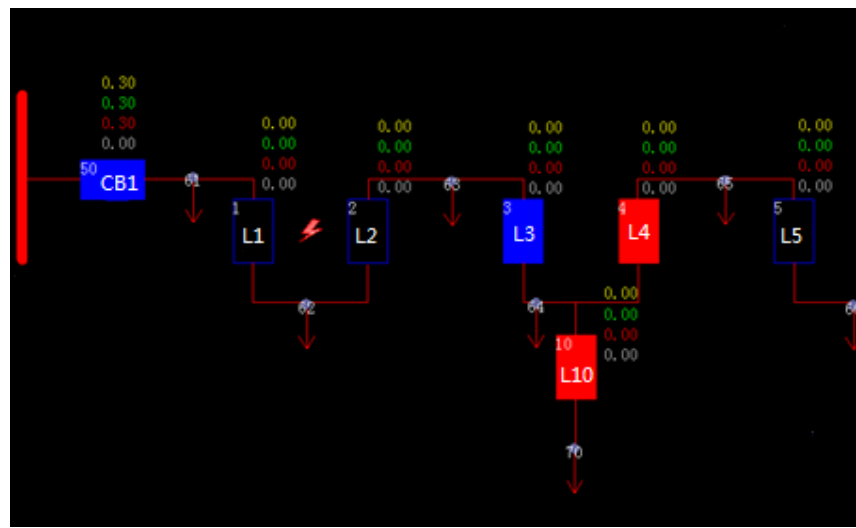

(b) Fault isolation.

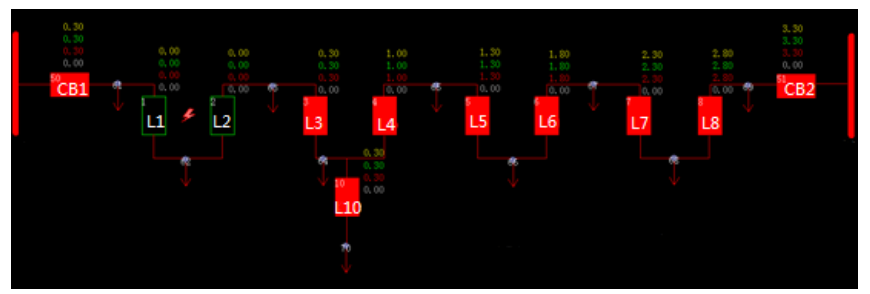

(c) Fault restoration.

Figure 3. Sufficient load transfer margin case.

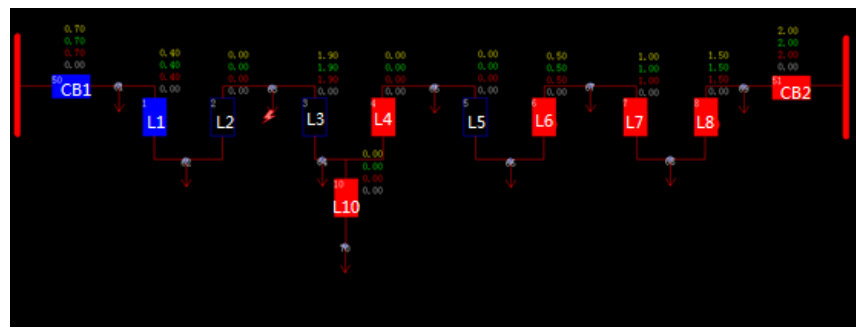

Figure 4. Insufficient load transfer margin case.

\section{Conclusion}

With the current DAS lack of comprehensive testing method, this paper proposes a fault simulation test method and establishes fault process efficiency evaluation index system. Typical distribution networks are designed and classified in five divisions of power supply areas, which makes testing scenarios more practical. Add more active interference into test case to meet the diversity and extensibility of test simulation. An evaluation method with several indexes of fault process efficiency is also proposed in this paper. The index is not only based on the result of fault handling, but also tracks the whole action process of fault isolation and restoration. In the test report, power recovery rate is provided to evaluate the load transfer capability in the end.

\section{Acknowledgment}

This work was supported in part by Grant 5442PD140007 from China Electric Power Research Institute and Grant 2014AA051901 from State Grid Corporation of China in Beijing.

\section{References}

1. W.G. Yang, M. Wen, Z.P. Zheng, Distribution Network and Automation (China: Water \& Power, 2004)

2. D. Liu, L. Sheng, AEPS, 25, 40-44(2001)

3. J. Liu, S.R. Zhao, X.Q. Zhang, AEPS, 39, 6-10 (2012)

4. D. Liu, Distribution Automation System Test (China: Water \& Power, 2004)

5. B.Y. Xu, T.Y. Li, AEPS, 34, 81-86 (2010)

6. D. Liu, Z.H. Ding, L.T. Teng, APES, 28 (2004)

7. W.X. Li, P. Wang, Z.M. Li, Y.C. Liu, IEEE Trans. on Power Delivery, 19, 2, 753-758 (2004)

8. Z.H. Weng, D. Liu, J.S. Liu, Y.H. Huang, AEPS, 33, 43-46 (2009)

9. W.S. Ling, D. Liu, X. Chen, AEPS, 36, 71-75 (2012)

10. J. Liu, X.Q. Zhang, S.R. Zhao, AEPS, 36, 67-71 (2012)

11. H.L. Cheng, K.C. Tang, J. Liu, HVE, 33, 166-172 (2007)

12. S.X. Wang, C.S. Wang, Modern distribution system analysis (China: Higher Education, 2014) 